\title{
RAPID SOLUTION OF PARAMETER-DEPENDENT LINEAR SYSTEMS FOR ELECTROMAGNETIC PROBLEMS IN THE FREQUENCY DOMAIN*
}

\author{
Martin Nilsson $^{\dagger}$
}

\begin{abstract}
The Minimal Residual Interpolation method reduces the number of iterations in an iterative method for multiple right hand sides. It uses computed solutions to initialize an iterative solver with an accurate guess. This paper demonstrates the efficiency of the method for frequency sweeps and solving scattering problems by plane waves incident from multiple angles. A bound on the number of solutions required for plane wave scattering before the remaining solutions are obtained by Minimal Residual Interpolation only is given. We discuss the performance of the method compared to iterative seed techniques. In a numerical example a reduction factor of 60 is obtained on the number of matrix vector multiplications.
\end{abstract}

Keywords: system of linear equations, multiple right hand sides, frequency sweeps, iterative method, method of moments, frequency domain, Maxwell's equations

AMS subject classification (MSC2000): 65F10, 65R20, 78M05

\section{Introduction}

The Method of Moments is a method for discretizing an integral equation into a dense system of linear equations. Since the system matrix is dense, a direct method like Gaussian elimination requires $\mathcal{O}\left(N^{3}\right)$ arithmetic operations, where $N$ is the number of unknowns. An iterative method requires $\mathcal{O}\left(M N^{2}\right)$ arithmetic

*Financial support has been obtained from Parallel and Scientific Computing Institute (PSCI), which is a competence center financed by Vinnova, The Swedish Agency for Innovation Systems, and the Swedish National Aeronautical Research Program, NFFP.

${ }^{\dagger}$ Department of Information Technology, Scientific Computing, Uppsala University, SE75105 Uppsala, Sweden (martin@it.uu.se). 
operations, where $M$ is the number of iterations. If $M$ is large, an iterative method can be as expensive as a direct method.

The Multilevel Fast Multipole Algorithm [14] (MLFMA) can compute the dense matrix vector multiplication in $\mathcal{O}(N \log N)$, reducing the work in an iterative solver to $\mathcal{O}(M N \log N)$. This makes MLFMA faster than a direct method. For a large number of right hand sides $K$ the advantage diminishes. The reason is that the additional work with Gaussian elimination is $\mathcal{O}\left(K N^{2}\right)$, while the work in MLFMA is $\mathcal{O}(K M N \log N)$.

In [6] the Minimal Residual Interpolation (MRI) method was introduced for systems with many right hand sides depending smoothly on a parameter. It reduces the work in any iterative solver by computing initial guesses to unsolved equations through least squares interpolation. Because of its relation to interpolation it can in many cases compute a solution that is as accurate as required. Since this reduces $K M$, the total work in an iterative solver is reduced.

This paper follows up on several of the issues in [6]. An extension of the algorithm to the case of a parameter dependent matrix was suggested in [6]. This is implemented and tested in this paper. A theorem relating the residual to an interpolation error is proved. It is an extension of a theorem in [6]. In this paper the method is used for computing the solutions to scattering problems by a plane wave at multiple frequencies. The case of scattering from plane waves that are incident from different angles is also considered. A bound on the number of solutions needed before MRI accurately computes the remaining solutions is given. Experiments demonstrate that MRI in both the angle and the frequency parameter is more effective than applying it in one of the parameters separately. Finally, MRI is compared with a version of the iterative seed method in [12].

The remaining sections of this paper are organized as follows. In the next section the algorithm is described. Then the convergence theorem is proved. The third section compares MRI and iterative seed methods. Next the Method of Moments is described and the number of solutions required for multiple incident plane waves is analyzed. Numerical experiments demonstrate the performance of the method. In one of the experiments a reduction factor of 60 is obtained on the number of matrix vector multiplications. The norm in the paper is the Euclidean vector norm and its subordinate spectral matrix norm. Vectors and matrices are typeset with a bold font.

\section{Minimal Residual Interpolation}

The Minimal Residual Interpolation method is an algorithm for solving multiple linear systems of equations that are smoothly dependent on one or several parameters. Assuming that the system matrix and right hand sides depend smoothly on a parameter an accurate initial guess for an iterative method is computed. 
The system of linear equations to be solved are

$$
\mathbf{A}_{i} \tilde{\mathbf{x}}_{i}=\mathbf{b}_{i}, i=1 \ldots M, \mathbf{A}_{j} \in \mathbb{C}^{N \times N}, \mathbf{x}_{i}, \mathbf{b}_{i}, \in \mathbb{C}^{N} .
$$

If a solution $\mathbf{x}_{i} \approx \tilde{\mathbf{x}}_{i}$ is obtained from an iterative method, e.g. GMRES, QMR, etc, the residual is given by

$$
\mathbf{r}_{i}=\mathbf{b}_{i}-\mathbf{A}_{i} \mathbf{x}_{i}
$$

Usually the solution satisfies a convergence criterion $\left\|\mathbf{r}_{i}\right\| \leq \varepsilon$ for some given $\varepsilon$.

Assume that the solutions to $m<M$ equations are known to precision given by the residual and that the solutions are linearly independent. Let $\mathbf{s}_{i, m+1}$ be defined by

$$
\mathbf{s}_{i, m+1}=\mathbf{A}_{m+1} \mathbf{x}_{i}=\mathbf{b}_{i}-\mathbf{r}_{i}+\left(\mathbf{A}_{m+1}-\mathbf{A}_{i}\right) \mathbf{x}_{i}, i=1 \ldots m
$$

and define

$$
\mathbf{X}_{m}=\left[\begin{array}{llll}
\mathbf{x}_{1} \mathbf{x}_{2} \ldots \mathbf{x}_{m}
\end{array}\right], \mathbf{S}_{m, m+1}=\left[\mathbf{s}_{1, m+1} \mathbf{s}_{2, m+1} \ldots \mathbf{s}_{m, m+1}\right] .
$$

Then an initial guess to equation $(m+1, k)$ can be computed as a linear combination of the previous solutions $\mathbf{x}_{m+1}=\mathbf{X}_{m} \mathbf{y}_{m}$. The coefficients $\mathbf{y}_{m}$ are given by the least squares solution $\mathbf{y}_{m}=\mathbf{R}_{\mathbf{S}_{m, m+1}}^{-1} \mathbf{Q}_{\mathbf{S}_{m, m+1}}^{H} \mathbf{b}_{m+1}$, where the QRfactorization of $\mathbf{S}_{m, m+1}$ is $\mathbf{S}_{m, m+1}=\mathbf{Q}_{\mathbf{S}_{m, m+1}} \mathbf{R}_{\mathbf{S}_{m, m+1}}$, and the initial guess is

$$
\mathbf{x}_{m+1}^{(0)}=\mathbf{X}_{m} \mathbf{R}_{\mathbf{S}_{m, m+1}}^{-1} \mathbf{Q}_{\mathbf{S}_{m, m+1}}^{H} \mathbf{b}_{m+1} .
$$

The residual for the initial guess $\mathbf{x}_{m+1}^{(0)}$ in (2) is

$$
\mathbf{r}_{m+1}^{(0)}=\mathbf{b}_{m+1}-\mathbf{A}_{m+1} \mathbf{X}_{m} \mathbf{y}_{m}=\left(\mathbf{I}-\mathbf{Q}_{\mathbf{S}_{m, m+1}} \mathbf{Q}_{\mathbf{S}_{m, m+1}}^{H}\right) \mathbf{b}_{m+1} .
$$

This expression for $\mathbf{r}_{m+1}^{(0)}$ is cheap to evaluate since $m \ll N$ and $\mathbf{Q}_{\mathbf{S}_{m, m+1}}^{H} \mathbf{b}_{m+1}$ is already computed in (2). The residual is small if $\mathbf{b}_{m+1}$ is almost spanned by $\mathbf{S}_{m, m+1}$, which is the case if $\mathbf{A}_{i}$ and $\mathbf{b}_{i}$ depend in a smooth way on a parameter $\phi_{i}$ and the difference $\Delta \phi=\phi_{i+1}-\phi_{i}$ is small, as we show in the next section. If $\left\|\mathbf{r}_{m+1}^{(0)}\right\|>\varepsilon$ then the initial guess $\mathbf{x}_{m+1}^{(0)}$ has to be improved by the iterative method. If $\left\|\mathbf{r}_{m+1}^{(k)}\right\| \leq \varepsilon_{I}$ for an $\varepsilon_{I} \leq \varepsilon$ then the iterations are interrupted and $\mathbf{s}_{m+1}^{(k)}$ is included in the basis $\mathbf{S}_{m, m+1}$ if

$$
\left\|\left(\mathbf{I}-\mathbf{Q}_{\mathbf{S}_{m, m+1}} \mathbf{Q}_{\mathbf{S}_{m, m+1}}^{H}\right) \mathbf{s}_{m+1}^{(k)}\right\|>\varepsilon_{s},
$$

where $\varepsilon_{s}>\varepsilon+\varepsilon_{I}$. Otherwise, $\mathbf{s}_{m+1}^{(k)}$ is almost linearly dependent of the columns of $\mathbf{S}_{m, m+1}$ which gives ill-conditioned matrices. This is particularly the case when $\mathbf{x}_{m+1}=\mathbf{x}_{m+1}^{(0)}$ and no iterations are necessary. Once the solution is found and (4) is satisfied we can construct $\mathbf{X}_{m+1}$ and $\mathbf{S}_{m+1, m+2}$. 
If it is known how $\mathbf{A}$ and $\mathbf{b}$ depend on the parameter $\phi$, an efficient order to solve the equations is indicated by Figure 1. In this case MRI solves the problems level by level in a binary tree, as explained in [6]. The black circles indicate the solutions that are obtained on the current level. A white circle indicate that the solution has been obtained on a previous level and is used to interpolate the remaining solutions using MRI. On each level the distance between two equations $\Delta \phi_{l}$ is reduced.
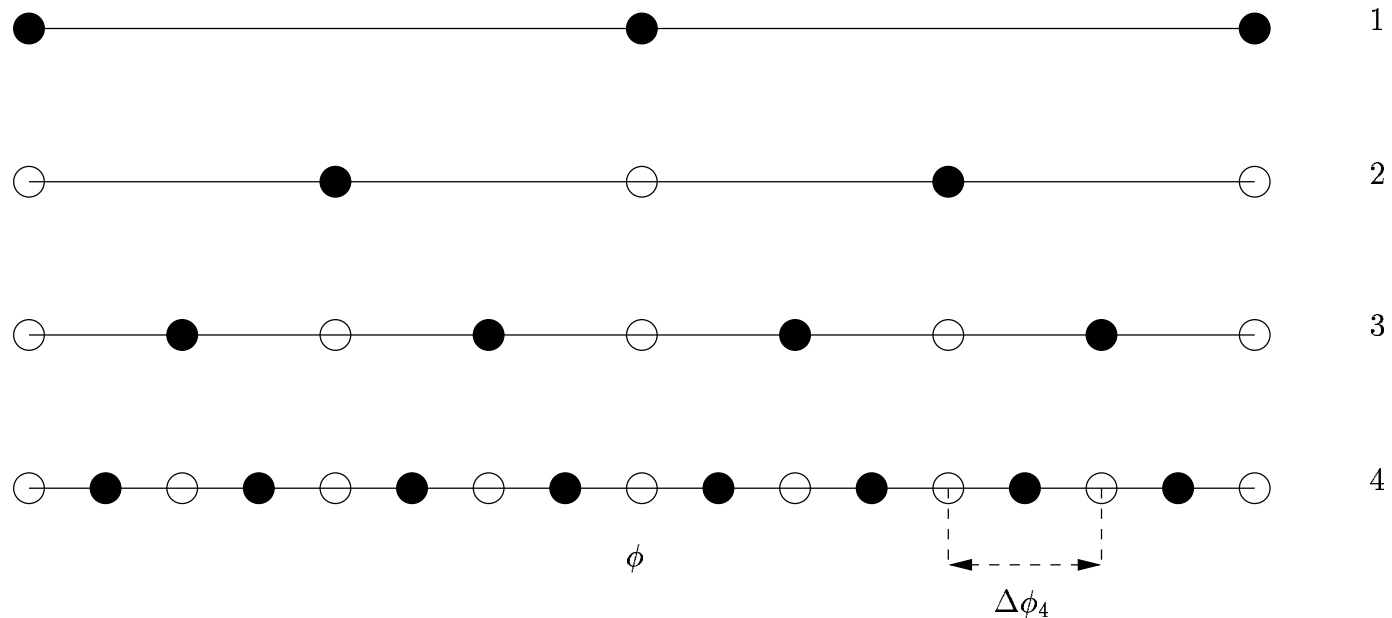

Figure 1: The order to solve the equations.

\section{Convergence properties}

A theorem relating the residual of the initial guess to an interpolation error for the case of constant matrix A was proved in [6]. Here, we state a similar theorem for the case when $\mathbf{A}$ also depends on $\phi$

\section{Theorem 1}

Assume that the components in the right hand side vectors $\mathbf{b}_{i}=\mathbf{b}\left(\phi_{i}\right)$ and the matrices $\mathbf{A}_{i}=\mathbf{A}\left(\phi_{i}\right)$ have $p$ continuous derivatives in $\phi$ and let $\mathbf{A}=\mathbf{A}(\phi)$ and $\mathbf{b}=\mathbf{b}(\phi),\left\|\mathbf{r}_{i}\right\| \leq \varepsilon_{I}$, $|\operatorname{det}(\mathbf{A}(\phi))| \geq C>0$ for some constant $C$ and $\phi_{\min } \leq \phi \leq \phi_{\max }$, and that an approximation to $\mathbf{b}_{\alpha}$ at $\phi_{\alpha}$ is computed by the minimization

$$
\min _{\mathbf{y}}\left\|\mathbf{b}_{\alpha}-\sum_{i=1}^{p} \mathbf{s}_{i, \alpha} y_{i}\right\| .
$$


Then

$$
\begin{aligned}
\left\|\mathbf{b}_{\alpha}-\sum_{i=1}^{p} \mathbf{s}_{i, \alpha} y_{i}\right\| & \leq \min \left(\sqrt{N}\left(\mathbf{A}_{\alpha} \mathbf{A}^{-1} \mathbf{b}\right)_{\max }^{(p)} \Delta \phi_{l-1}^{p}\right. \\
& \left.+\sqrt{p}\|\mathbf{l}\| \max \left\|\mathbf{A}_{\alpha} \mathbf{A}^{-1}\right\| \varepsilon_{I},\left\|\mathbf{b}_{\alpha}\right\|\right)
\end{aligned}
$$

where $\left(\mathbf{A}_{\alpha} \mathbf{A}^{-1} \mathbf{b}\right)_{\max }^{(p)}=\max _{i} \max _{\phi}\left|\sum_{j=1}^{N}\left(A_{\alpha}\right)_{i j} \tilde{x}_{j}^{(p)}(\phi)\right|, \tilde{x}_{j}^{(p)}$ is the $p$ :th derivative of $\tilde{x}_{j}=D_{j} / D$ where $D=\operatorname{det}(\mathbf{A})$ and $D_{j}$ is the determinant of the matrix with the $j$ :th column of $\mathbf{A}$ replaced by $\mathbf{b}$, and $\mathbf{l}$ consists of the coefficients of the Lagrange polynomial at the point $\phi_{\alpha}$.

Proof

Let $\gamma_{i}$ be a set of coefficients different from $y_{i}$ and $\mathbf{x}_{i}=\mathbf{x}\left(\phi_{i}\right)$. The triangle inequality together with the equality $\mathbf{s}_{i}=\mathbf{A}_{\alpha} \mathbf{x}_{i}=\mathbf{A}_{\alpha} \mathbf{A}_{i}^{-1}\left(\mathbf{b}_{i}-\mathbf{r}_{i}\right)$ yields

$$
\begin{aligned}
\left\|\mathbf{b}_{\alpha}-\sum_{i=1}^{p} \mathbf{s}_{i} y_{i}\right\| & \leq\left\|\mathbf{b}_{\alpha}-\sum_{i=1}^{p} \mathbf{s}_{i} \gamma_{i}\right\|=\left\|\mathbf{A}_{\alpha} \mathbf{A}_{\alpha}^{-1} \mathbf{b}_{\alpha}-\sum_{i=1}^{p} \mathbf{A}_{\alpha} \mathbf{A}_{i}^{-1}\left(\mathbf{b}_{i}-\mathbf{r}_{i}\right) \gamma_{i}\right\| \\
& \leq\left\|\mathbf{A}_{\alpha} \mathbf{A}_{\alpha}^{-1} \mathbf{b}_{\alpha}-\sum_{i=1}^{p} \mathbf{A}_{\alpha} \mathbf{A}_{i}^{-1} \mathbf{b}_{i} \gamma_{i}\right\|+\left\|\sum_{i=1}^{p} \mathbf{A}_{\alpha} \mathbf{A}_{i}^{-1} \mathbf{r}_{i} \gamma_{i}\right\| .
\end{aligned}
$$

To obtain an estimate of the first part we let $l(\phi)$ be the interpolating polynomial of degree less than $p$ of the $j$ :th component of $\mathbf{A}_{\alpha} \mathbf{A}^{-1} \mathbf{b}$ through the points $\phi_{i}, i=1 \ldots p$. Cramer's rule gives $\tilde{x}_{j}(\phi)=D_{j}(\phi) / D(\phi)$. Note that $\tilde{x}_{j}(\phi)$ is a function with $p$ continuous derivatives of $\phi$. Since $D \geq C$ by assumption and the elements of $\mathbf{A}(\phi)$ and $\mathbf{b}(\phi)$ have $p$ continuous derivatives, the Leibnitz' rule applied to the product and sums of the determinants give a function $\tilde{x}_{j}$ with $p$ continuous derivatives. The denominator causes no problem since derivation only gives higher powers of $D$ in the denominator. By an interpolation theorem [2] we have for all $i$

$$
\begin{aligned}
\left|\sum_{j=1}^{N} A_{i j}\left(\phi_{\alpha}\right) \tilde{x}_{j}\left(\phi_{\alpha}\right)-l\left(\phi_{\alpha}\right)\right| & =\left|\sum_{j=1}^{N} A_{i j}\left(\phi_{\alpha}\right) \tilde{x}_{j}\left(\phi_{\alpha}\right)-\sum_{k=1}^{p} \sum_{j=1}^{N} A_{i j}\left(\phi_{\alpha}\right) \tilde{x}_{j}\left(\phi_{k}\right) l_{\alpha k}\right| \\
& \leq\left|\left(\sum_{j=1}^{N} A_{i j}\left(\phi_{\alpha}\right) \tilde{x}_{j}\right)^{(p)}\right|\left|W_{\alpha}\right| / p ! .
\end{aligned}
$$

where $\left|\left(\sum_{j=1}^{N} A_{i j}\left(\phi_{\alpha}\right) \tilde{x}_{j}\right)^{(p)}\right|=\max _{\phi}\left|\left(\sum_{j=1}^{N} A_{i j}\left(\phi_{\alpha}\right) \tilde{x}_{j}(\phi)\right)^{(p)}\right|$, the function $W_{\alpha}=\prod_{i=1}^{p}\left(\phi_{\alpha}-\phi_{i}\right)$, and $l_{\alpha k}$ are the coefficients of the Lagrange polynomial. 
Thus, if $\gamma_{k}=l_{\alpha k}$ then

$$
\begin{aligned}
\| \mathbf{A}_{\alpha} \mathbf{A}_{\alpha}^{-1} \mathbf{b}_{\alpha} & -\sum_{k=1}^{p} \mathbf{A}_{\alpha} \mathbf{A}_{k}^{-1} \mathbf{b}_{k} l_{\alpha k} \|^{2} \\
& =\sum_{i=1}^{N}\left|\sum_{j=1}^{N}\left(A_{\alpha}\right)_{i j} \tilde{x}_{j}\left(\phi_{\alpha}\right)-\sum_{k=1}^{p} \sum_{j=1}^{N}\left(A_{\alpha}\right)_{i j} \tilde{x}_{j}\left(\phi_{k}\right) l_{\alpha k}\right|^{2} \\
& \leq \sum_{i=1}^{N}\left|\left(\sum_{j=1}^{N} A_{i j}\left(\phi_{\alpha}\right) \tilde{x}_{j}\right)^{(p)} W_{\alpha} / p !\right|^{2} \\
& =\left(W_{\alpha} / p !\right)^{2} \sum_{i=1}^{N}\left|\left(\sum_{j=1}^{N} A_{i j}\left(\phi_{\alpha}\right) \tilde{x}_{j}\right)^{(p)}\right|^{2} \\
& \leq\left(W_{\alpha} / p !\right)^{2} N\left(\left(\mathbf{A}_{\alpha} \mathbf{A}^{-1} \mathbf{b}\right)_{\max }^{(p)}\right)^{2} .
\end{aligned}
$$

Since $\phi_{\alpha} \in\left[\phi_{1}, \phi_{M}\right]$, an upper bound on $\left|W_{\alpha}\right| / p$ ! is $\Delta \phi_{l-1}^{p}$.

With $\mathbf{R}=\left[\left(\mathbf{A}_{\alpha} \mathbf{A}_{1}^{-1} \mathbf{r}_{1}\right) \ldots\left(\mathbf{A}_{\alpha} \mathbf{A}_{p}^{-1} \mathbf{r}_{p}\right)\right] \in \mathbb{C}^{N \times p}$, the choice of Lagrange coefficients in the first part together with Cauchy-Schwartz' inequality gives the estimate of the second part since

$$
\begin{aligned}
\left\|\sum_{k=1}^{p}\left(\mathbf{A}_{\alpha} \mathbf{A}_{k}^{-1} \mathbf{r}_{k}\right) l_{\alpha k}\right\|^{2} & =\sum_{j=1}^{N}\left|\mathbf{R}_{j,:}\right|^{2} \leq \sum_{j=1}^{N}\|\mathbf{l}\|^{2}\left\|\mathbf{R}_{j,:}\right\|^{2} \\
& \leq\|\mathbf{l}\|^{2} \sum_{k=1}^{p} \sum_{j=1}^{N}\left|R_{j k}\right|^{2} \\
& \leq p\|\mathbf{l}\|^{2} \max _{k}\left\|\mathbf{A}_{\alpha} \mathbf{A}_{k}^{-1} \mathbf{r}_{k}\right\|^{2} \\
& \leq p\|\mathbf{l}\|^{2} \max _{k}\left\|\mathbf{A}_{\alpha} \mathbf{A}_{k}^{-1}\right\|^{2} \varepsilon_{I}^{2}
\end{aligned}
$$

An upper bound of the error in the approximation is given by $y_{i}=0$ for all $i$.

Compared to simple interpolation, the advantage is that the second error term is kept under control, which is not guaranteed by interpolation as indicated above. The reason is that the Lagrange coefficients are not optimal in the $l_{2}$-norm. Thus, the second part can not be controlled by interpolation.

\section{Comparison with iterative seed methods}

Iterative seed methods are methods that solve for multiple right hand sides [5, 12]. The idea is to solve iteratively one or several of the equations and use the vectors spanning the Krylov subspace generated in the solution process to update the remaining equations. The assumption is that if the solutions are close to each other the residuals of the remaining equations will go down together with the residuals of the equations that are solved. Here, we will analyze a seed method based on block GMRES, but the conclusions carry over to other iterative seed methods as well.

Assume that block GMRES without deflation is used to solve (1) and that $\mathbf{A}_{i}=\mathbf{A}$ is independent of $i$. Let $m<M$ be the number of right hand sides 
that are solved for in block GMRES. Let $m$ be so large that the solutions to the remaining equations can be computed by MRI with residual $\left\|\mathbf{r}_{l}^{(0)}\right\|=\| \mathbf{b}_{l}-$ $\mathbf{S}_{m} \mathbf{y}_{m} \| \leq \varepsilon_{I}$, where $\mathbf{S}_{m}=\mathbf{A} \mathbf{X}_{m}$. Then if the Arnoldi vectors that were produced by block GMRES are used to compute solutions to the remaining equations in a seed iterative way the residual is $\leq \varepsilon_{I}$. The reason is that the $(q+1)$ :th step of the Arnoldi process generates an orthonormal basis $\mathbf{V}_{m q} \in \mathbb{C}^{N \times m q}$ for $\mathbf{X}_{m}$ such that

$$
\mathbf{A} \mathbf{V}_{m q}=\mathbf{V}_{m(q+1)} \mathbf{H}_{m(q+1) \times m q}, \mathbf{X}_{m}=\mathbf{V}_{m q} \mathbf{Z}_{m},
$$

where $\mathbf{H}_{m(q+1) \times m q} \in \mathbb{C}^{m(q+1) \times m q}$ is an upper block Hessenberg matrix, $\mathbf{V}_{m(q+1)}=$ $\left[\mathbf{V}_{m q}\left(\mathbf{v}_{q+1}\right)_{m}\right] \in \mathbb{C}^{N \times m(q+1)}$ is another orthonormal matrix, and $\mathbf{Z}_{m} \in \mathbb{C}^{m q \times m}$. It follows that

$$
\mathbf{S}_{m}=\mathbf{A} \mathbf{X}_{m}=\mathbf{A} \mathbf{V}_{m q} \mathbf{Z}_{m}=\mathbf{V}_{m(q+1)} \mathbf{H}_{m(q+1) \times m q} \mathbf{Z}_{m}
$$

That is $\mathbf{S}_{m}$ is spanned by a subspace of $\mathbf{V}_{m(q+1)}$. Thus, the Krylov subspace spanned by $\mathbf{V}_{m(q+1)}$ can be used to compute the remaining solutions by the GMRES process.

The drawbacks with this method is that the size of the space $\mathbf{V}_{m(q+1)}$ will be larger than the size of $\mathbf{S}_{m}$ and also no restarts are allowed for the conclusion to hold. It is expected that block GMRES with restarts can be used but that would require that all right hand sides are stored in memory so that they can be updated during the restart process. On the other hand MRI stores the computed solutions and the vectors $\mathbf{S}_{m}$. The vectors could have been computed by a single right hand side solver, block GMRES or any other method.

Let us assume that the right hand sides can be computed when they are needed. Since the update of $\mathbf{S}_{m}$ is carried out after the iterations are finished the memory requirements are $\mathcal{O}(m)$ instead of $\mathcal{O}(M)$. Instead of GMRES one could have used a short term recurrence method like the block QMR method in [5]. The solution is then updated step by step from a recurrence formula. Only a few recurrence vectors are stored at a time. Since all recurrence vectors are needed for the Krylov subspace that span $\mathbf{S}_{m}$, all solution vectors must be updated at the same time by the recurrence in order for the conclusion to hold. This also yields a memory requirement $\mathcal{O}(M)$.

\section{Method of Moments}

Consider the time-harmonic electromagnetic scattering from a perfect electric conductor (PEC). Combining the Electric Field Integral Equation (EFIE) and the Magnetic Field Integral Equation (MFIE) in variational form yields the Combined Field Integral Equation (CFIE) [9] 


$$
\begin{aligned}
& \alpha \int_{\Gamma} \int_{\Gamma} G\left(\mathbf{x}, \mathbf{x}^{\prime}\right)\left(\mathbf{J} \cdot \mathbf{J}^{\prime}-\frac{1}{\kappa^{2}} \nabla_{\Gamma} \cdot \mathbf{J} \nabla_{\Gamma} \cdot \mathbf{J}^{\prime}\right) d \Gamma d \Gamma \\
& +(1-\alpha) \frac{\imath}{\kappa} \int_{\Gamma} \hat{\mathbf{n}} \times \int_{\Gamma} \nabla_{\mathbf{x}^{\prime}} G\left(\mathbf{x}, \mathbf{x}^{\prime}\right) \times \mathbf{J} \cdot \mathbf{J}^{\prime} d \Gamma d \Gamma \\
& =-\alpha \frac{1}{\imath \kappa Z} \int_{\Gamma} \mathbf{E}_{a} \cdot \mathbf{J}^{\prime} d \Gamma+(1-\alpha) \frac{\imath}{\kappa} \int_{\Gamma} \hat{\mathbf{n}} \times \mathbf{H}_{a} \cdot \mathbf{J}^{\prime} d \Gamma .
\end{aligned}
$$

Here, $\mathbf{J}$ is the unknown electric current on the surface $\Gamma$ of the scatter, $\mathbf{J}^{\prime}$ is the test current, $\kappa$ is the wavenumber, $Z$ is the impedance in free space, $\hat{\mathbf{n}}$ is the unit normal pointing outward from $\Gamma$, and $\imath=\sqrt{-1}$. The function $G\left(\mathbf{x}, \mathbf{x}^{\prime}\right)$ is the free-space Green's function for Helmholtz' equation. The parameter $\alpha$ can vary between 0 (MFIE) and 1 (EFIE). The right hand side depends on the applied electric field $\mathbf{E}_{a}$ and the applied magnetic field $\mathbf{H}_{a}$.

Galerkins method with RWG basis functions are used for discretization [10]. The discretization leads to a dense, complex system of equations of the form (1). The unknowns in $\mathbf{x}$ are the coefficients for each basis function and the right hand side $\mathbf{b}$ depends on the applied fields $\mathbf{E}_{a}$ and $\mathbf{H}_{a}$. Only the right hand side is changed, if the wavenumber is constant and the applied fields are changed. A change of wavenumber affects the right hand side and the matrix $\mathbf{A}$. The matrix A has continous derivatives with respect to $\kappa$, but is singular when $\alpha=0$ or $\alpha=1$ and the wavenumber corresponds to a nullspace solution.

The applied electric and magnetic fields in (6) at $\mathbf{x}$ can be written

$$
\mathbf{E}_{a}\left(\mathbf{x}, \hat{\boldsymbol{\kappa}}_{a}\right)=\mathbf{E}_{0} \exp \left(-\imath \kappa \hat{\boldsymbol{\kappa}}_{a} \cdot \mathbf{x}\right), \mathbf{H}_{a}\left(\mathbf{x}, \hat{\boldsymbol{\kappa}}_{a}\right)=\mathbf{H}_{0} \exp \left(-\imath \kappa \hat{\boldsymbol{\kappa}}_{a} \cdot \mathbf{x}\right)
$$

for a plane wave traveling in the direction given by the unit vector $\hat{\boldsymbol{\kappa}}_{a}$. Discretization with the test functions $\mathbf{j}_{j}$ and approximation of the integral with a quadrature rule with $q$ positive weights $w_{j k}$ gives the $j$ :th component of the discretized right hand side

$$
b_{j}\left(\hat{\boldsymbol{\kappa}}_{a}\right)=\sum_{k=1}^{q} w_{j k} \mathbf{K}\left(\hat{\boldsymbol{\kappa}}_{a}\right) \cdot \mathbf{j}_{j}\left(\mathbf{x}_{j k}\right) \exp \left(-\imath \kappa \hat{\boldsymbol{\kappa}}_{a} \cdot \mathbf{x}_{j k}\right)
$$

where $\mathbf{K}\left(\hat{\boldsymbol{\kappa}}_{a}\right)$ depends on $\mathbf{E}_{0}$ and $\mathbf{H}_{0}$.

The GMRES method [11] with the fast multipole method [4] for the matrixvector multiplications is used to solve the system of equations. The major cost in the iterations is the multiplication of an arbitrary vector by the matrix. The matrix is preconditioned with a modified Sparse Approximate Inverse Preconditioner (SPAI) as in [7], [8], which improves the convergence rate especially for EFIE. For several right hand sides, the block version of GMRES accelerates the convergence as in [13]. 
In the Fast Multipole Method a truncated series is used to approximate the Green's function $G\left(\mathbf{x}, \mathbf{x}^{\prime}\right)$. The number of terms needed for the approximation of the Green's function varies with wave number and thus frequency as [3]

$$
L \approx \kappa D+1.8\left(-\log _{10} \epsilon\right)^{\frac{2}{3}}(\kappa D)^{\frac{1}{3}}
$$

where $D$ is a problem dependent parameter that depends on a predetermined box size and $\epsilon$ is the required relative error in the approximation of the Green's function. In a frequency sweep, $L$ will vary according to (9). Thus, the use of the Fast Multipole Method implies that the system matrix is not infinitely smooth. This will affect the convergence rate of the Minimal Residual Interpolation method.

\subsection{Bounds on the number of solutions}

We consider the case of plane wave scattering with constant real wave number and derive approximate bounds on the number of right hand sides that need to be solved before MRI accurately predicts the solution to the remaining equations. To simplify the analysis we consider the case when

$$
\tilde{b}_{j}\left(\hat{\boldsymbol{\kappa}}_{a}\right)=\exp \left(-\imath \kappa \hat{\boldsymbol{\kappa}}_{a} \cdot \mathbf{x}_{j}\right)
$$

The bounds are derived from the expansion [1]

$$
e^{\imath \boldsymbol{\kappa} \cdot \mathbf{X}}=\sum_{l=0}^{\infty} \imath^{l}(2 l+1) j_{l}(X \kappa) P_{l}(\hat{\mathbf{X}} \cdot \hat{\boldsymbol{\kappa}})
$$

Here, $j_{l}(x)$ is the spherical Bessel function of order $l$ and $P_{l}(\cos \Theta)$ is the Legendre polynomial of order $l$, the absolute value of a vector is $x=|\mathbf{x}|$ and a unit length vector is denoted by a hat above it. The spherical Bessel function is linked to the Legendre polynomial by the relationship [4]

$$
4 \pi \imath^{l} j_{l}(X \kappa) P_{l}(\hat{\mathbf{X}} \cdot \hat{\boldsymbol{\kappa}})=\int_{\mathcal{S}} e^{\imath \boldsymbol{\kappa}_{s} \cdot \mathbf{X}} P_{l}\left(\hat{\boldsymbol{\kappa}}_{s} \cdot \hat{\boldsymbol{\kappa}}\right) d \hat{\boldsymbol{\kappa}}
$$

where the integration is taken over the unit sphere $\mathcal{S}$. Truncating (11) after $L$ terms and inserting (12) and changing the order of summation and integration yields

$$
\begin{aligned}
e^{\imath \boldsymbol{\kappa} \cdot \mathbf{X}} & \approx \sum_{l=0}^{L} \imath^{l}(2 l+1) j_{l}(X \kappa) P_{l}(\hat{\mathbf{X}} \cdot \hat{\boldsymbol{\kappa}}) \\
& =\frac{1}{4 \pi} \int_{\mathcal{S}} \sum_{l=0}^{L}(2 l+1) e^{\imath \boldsymbol{\kappa}_{s} \cdot \mathbf{X}} P_{l}\left(\hat{\boldsymbol{\kappa}}_{s} \cdot \hat{\boldsymbol{\kappa}}\right) d \hat{\boldsymbol{\kappa}}
\end{aligned}
$$

A quadrature formula that is exact for the $2 L$ first spherical harmonics is exact for the integral in $(13)$. In general $\mathcal{O}\left(L^{2}\right)$ nodes are needed. The trapezoidal rule 
with $2 L+1$ nodes in $\phi$ and Gauss-quadrature with $L+1$ nodes in $\theta$ is enough, but not optimal. Thus

$$
e^{\imath \boldsymbol{\kappa} \cdot \mathbf{X}} \approx \sum_{k=1}^{K} e^{\imath \boldsymbol{\kappa}_{k} \cdot \mathbf{X}} \sum_{l=0}^{L} \frac{w_{k}}{4 \pi}(2 l+1) P_{l}\left(\hat{\boldsymbol{\kappa}}_{k} \cdot \hat{\boldsymbol{\kappa}}\right)
$$

where $w_{k}$ is the the weight associated with node $\hat{\boldsymbol{\kappa}}_{k}$ and $K=\mathcal{O}\left(L^{2}\right)$. If equation (14) is inserted into (8) one has

$$
\tilde{\mathbf{b}}(\hat{\boldsymbol{\kappa}}) \approx \sum_{k=1}^{K} \tilde{\mathbf{b}}\left(\hat{\boldsymbol{\kappa}}_{k}\right) \sum_{l=0}^{L} \frac{w_{k}}{4 \pi}(2 l+1) P_{l}\left(\hat{\boldsymbol{\kappa}}_{k} \cdot \hat{\boldsymbol{\kappa}}\right)
$$

If $\kappa$ is real we have

$$
\|\tilde{\mathbf{r}}(\hat{\boldsymbol{\kappa}}) \mid=\| \tilde{\mathbf{b}}(\hat{\boldsymbol{\kappa}})-\sum_{k=1}^{K} \gamma_{k} \tilde{\mathbf{b}}\left(\hat{\boldsymbol{\kappa}}_{k}\right)\|=\| e^{\imath \boldsymbol{\kappa} \cdot \mathbf{X}_{c}} \tilde{\mathbf{b}}(\hat{\boldsymbol{\kappa}})-e^{\imath \boldsymbol{\kappa} \cdot \mathbf{X}_{c}} \sum_{k=1}^{K} \gamma_{k} \tilde{\mathbf{b}}\left(\hat{\boldsymbol{\kappa}}_{k}\right) \|
$$

where $\mathbf{X}_{c}$ is the center of the object. With the modified right hand side $e^{\imath \boldsymbol{\kappa} \cdot \mathbf{X}_{c}} \tilde{\mathbf{b}}(\hat{\boldsymbol{\kappa}})$ the largest argument of the spherical Hankel function is $\max _{\mathbf{X}} \kappa\left|\mathbf{X}-\mathbf{X}_{c}\right|$. Let $\gamma_{k}=\sum_{l=0}^{L} \frac{w_{k}}{4 \pi}(2 l+1) P_{l}\left(\hat{\boldsymbol{\kappa}}_{k} \cdot \hat{\boldsymbol{\kappa}}\right)$ and $R=\max _{\mathbf{X}}\left|\mathbf{X}-\mathbf{X}_{c}\right|$ be the radius of the smallest sphere enclosing the object. From (11) and (13) and the fact that $j_{l}(x)$ is a rapidly decreasing function for fixed $x$ and large $l>x$

$$
\begin{aligned}
\frac{\left|\tilde{r}_{j}(\hat{\boldsymbol{\kappa}})\right|}{\|\tilde{\mathbf{b}}(\hat{\boldsymbol{\kappa}})\|} \leq & \frac{\left|\sum_{l=L+1}^{\infty} \imath^{l}(2 l+1) j_{l}(X \kappa) P_{l}(\hat{\mathbf{X}} \cdot \hat{\boldsymbol{\kappa}})\right|}{\|\tilde{\mathbf{b}}(\hat{\boldsymbol{\kappa}})\|} \\
& \approx \frac{\left|(2 L+3) j_{L+1}(X \kappa) P_{L+1}(\hat{\mathbf{X}} \cdot \hat{\boldsymbol{\kappa}})\right|}{\|\tilde{\mathbf{b}}(\hat{\boldsymbol{\kappa}})\|} \leq \frac{\left|(2 L+3) j_{L+1}(X \kappa)\right|}{\|\tilde{\mathbf{b}}(\hat{\boldsymbol{\kappa}})\|}
\end{aligned}
$$

since $\left|P_{L+1}(\hat{\mathbf{X}} \cdot \hat{\kappa})\right| \leq 1$. If

$$
\frac{\left|\tilde{r}_{j}(\hat{\boldsymbol{\kappa}})\right|}{\|\tilde{\mathbf{b}}(\hat{\boldsymbol{\kappa}})\|} \leq \frac{\varepsilon_{I}}{\sqrt{N}}
$$

the residual $\|\tilde{\mathbf{r}}(\hat{\boldsymbol{\kappa}})\| \leq \varepsilon_{I}\|\tilde{\mathbf{b}}(\hat{\boldsymbol{\kappa}})\|$. The truncation number $L$ that is required for this to hold can be estimated. The worst case in (15) is when $X=R$. Using the asymptotic expansion for the spherical Bessel functions [3] and the arguments in [3] for the number of Fast Multipole terms, the number $L$ can be estimated as

$$
L \approx \kappa R+1.8\left(-\log _{10}\left(\frac{\varepsilon_{I}\|\tilde{\mathbf{b}}(\hat{\boldsymbol{\kappa}})\|}{\sqrt{N}}\right)\right)^{\frac{2}{3}}(\kappa R)^{\frac{1}{3}}
$$


By the assumption in (10) $\|\tilde{\mathbf{b}}(\hat{\boldsymbol{\kappa}})\|=\sqrt{N}$ so the final estimate is

$$
L \approx \kappa R+1.8\left(-\log _{10} \varepsilon_{I}\right)^{\frac{2}{3}}(\kappa R)^{\frac{1}{3}}
$$

independent of the number of unknowns.

Thus, when solutions over the entire sphere of plane wave directions are sought, $K=\mathcal{O}\left(L^{2}\right)$ solutions are needed before the remaining solutions are accurately predicted by interpolation. With equidistant points, spectral theory predicts that $K \approx 4 L^{2}$.

It seems reasonable to assume that when solutions in a plane of plane wave directions are sought only $K=\mathcal{O}(L)$ solutions are needed. This can be proved based on the observation that [1]

$$
e^{-\imath \kappa \hat{\boldsymbol{\kappa}}_{a} \cdot \mathbf{X}}=e^{-\imath \kappa X \cos (\alpha-\phi)}=\sum_{l=-\infty}^{\infty} \imath^{-l} J_{l}(\kappa X) e^{\imath l(\alpha-\phi)}
$$

in that case. Here, $J_{l}(x)$ is the Bessel function of order $l$. The same asymptotic expansion as before give the formula in (16). If equidistant points are used $K \approx 2 L$ from spectral theory.

The arguments used here should be applicable to (8) as well.

\section{Numerical experiments}

Numerical results are compared to the theoretical results obtained in the previous sections. The Fast Multipole accelerated Method of Moments code described in Section 5 is used for validation. In the experiments $\alpha=0.5$ is used in (6) and the iterative method is preconditioned with the Sparse Approximate Inverse preconditioner in [7]. As a test example a model aircraft called RUND depicted in Figure 2 is used. By measuring the largest and smallest coordinate in the $x$, $y$ and $z$ directions it is concluded that the aircraft fits into a sphere of radius at most 0.6 meters. In the experiments two frequency ranges are considered. One is around $1.5 \mathrm{GHz}$, where the geometry is resolved with $N=16218$ edges, and one is around $6 \mathrm{GHz}$, where the geometry is resolved with $N=64959$ edges.

The aim of the first experiment is to validate the predictions in Theorem 1. Two experiments on RUND are made. In both experiments scattering from a plane wave traveling in a direction towards the nose of the airplane is considered. The experiments predict the solution at $1.5 \mathrm{GHz}$ and $6 \mathrm{GHz}$ from solutions obtained at surrounding frequencies. In order to avoid the influence of the error in the iterative solver we choose $\epsilon=\epsilon_{I}=10^{-9}$. Figure 3 shows the relative residual of the initial guess $\left\|\mathbf{r}^{(0)}\right\| /\|\mathbf{b}\|$ as a function of the distance in frequency between the interpolation points $\Delta f$. The result for 2,4 and 8 interpolation points are shown. As the number of interpolation points is increased the convergence rate increases exponentially. The rate is not as fast as predicted by theory 


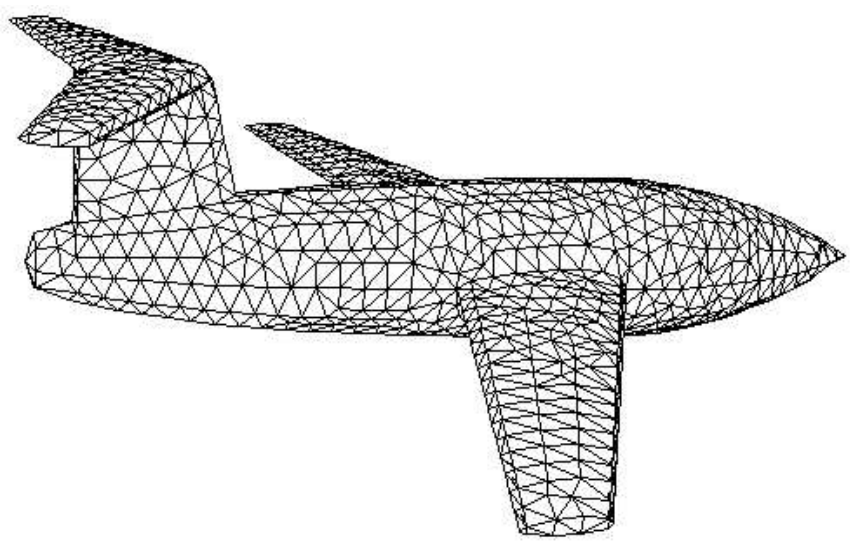

Figure 2: The generic aircraft model called RUND.

though. One possible reason could be that the Fast Multipole Method is used, which implies that the impedance matrix is not completely continuous as noted in Section 5.
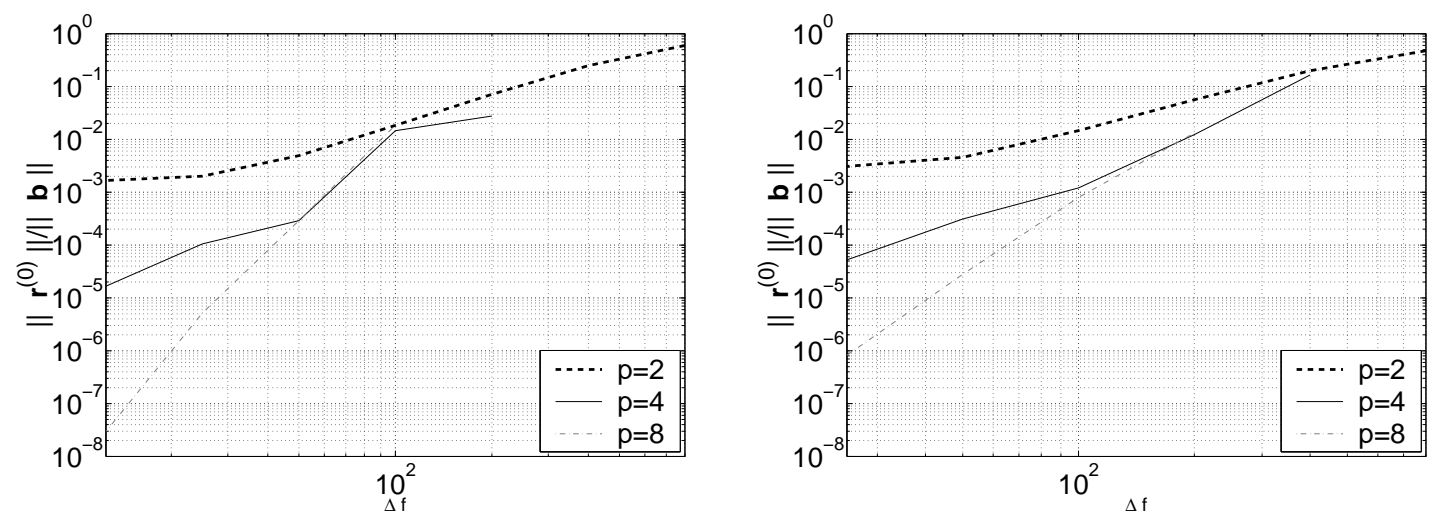

Figure 3: The relative residual of the initial guess as a function of $\Delta f$ for different number of interpolation vectors at $1.5 \mathrm{GHz}$ (left) and $6 \mathrm{GHz}$ (right).

In the second experiment the number of terms needed to accurately predict the remaining solutions as a function of $\epsilon$ is examined. The iterative solver uses $\epsilon_{I}=10^{-7}$ and the case of constant frequency and incident plane wave directions in a plane is considered. Figure 4 shows the predicted number of terms compared to the experimental number of terms at $1.5 \mathrm{GHz}$ and $6 \mathrm{GHz}$. In both cases the predicted number is larger than the actual number. One reason is that the predicted radius of the sphere is too large. The monostatic Radar Cross Section for the two cases is plotted in Figure 5. At $6 \mathrm{GHz}$ a comparison is made with the results obtained from a Method of Moments code using EFIE. The solutions are in close agreement.

The last experiment demonstrates that MRI can be used for interpolation 


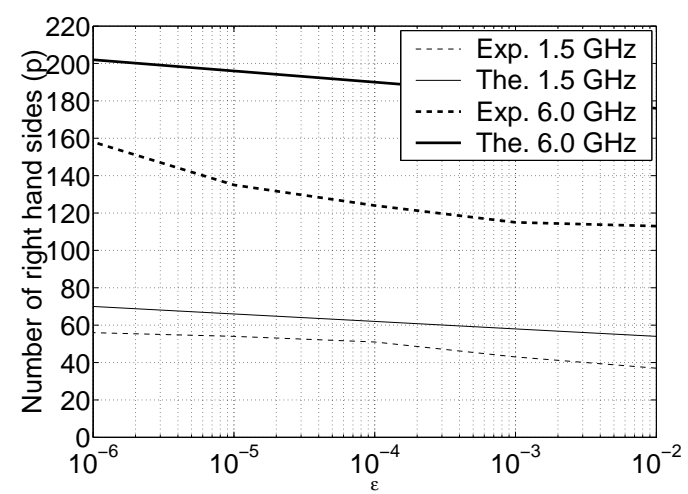

Figure 4: A comparison of the theoretical number of solutions and the number of solutions needed in the experiments.
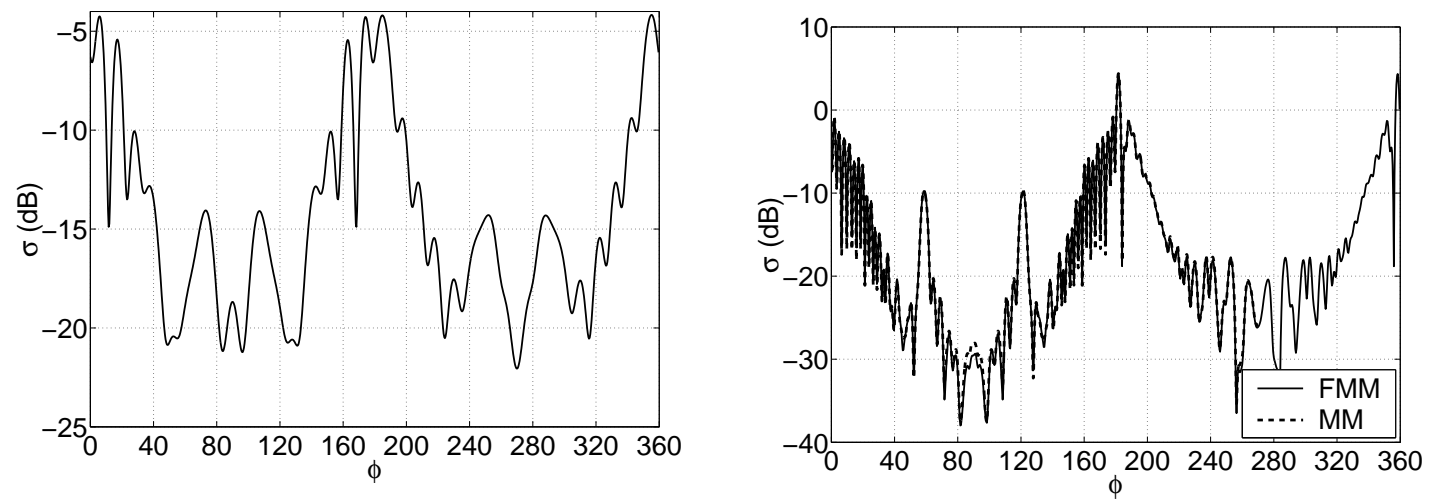

Figure 5: The monostatic Radar Cross Section at $1.5 \mathrm{GHz}$ (left) and $6 \mathrm{GHz}$ (right).

in both frequency and angle at the same time. In this case all the solutions at a fixed frequency are computed by MRI. Solutions that were used to predict the other solutions are kept and used in the interpolation at the next frequency. Both frequency and angle use the strategy in Figure 1 to pick the next parameter point. The frequency range is $1.45 \mathrm{GHz}$ to $1.65 \mathrm{GHz}$ sampled at 17 frequency points and the angle varies between 0 and 359.6 degrees sampled at 900 points. In the experiments $\epsilon=\epsilon_{I}=10^{-3}$ was used and the solver was full GMRES for one right hand side. Figure 6 plots the monostatic Radar Cross Section for the frequency range and Figure 7 shows the relative residual of the initial guess. The relative residual $10^{-3}$ is plotted for comparison in Figure 7. In MRI, 234 of the 15300 solutions was enough to predict the remaining solutions with MRI. The iterative solver computed 1790 matrix vector multiplications. In addition 3368 matrix vector multiplications were computed in the initialization step at the different frequencies. This should be compared to an estimated 291000 matrix vector multiplications if GMRES is used alone on each equation and a computed 11959 matrix vector multiplications if MRI is used to interpolate in the angle 
only. In the estimate it is assumed that on average each right hand side requires 19 iterations in full GMRES.

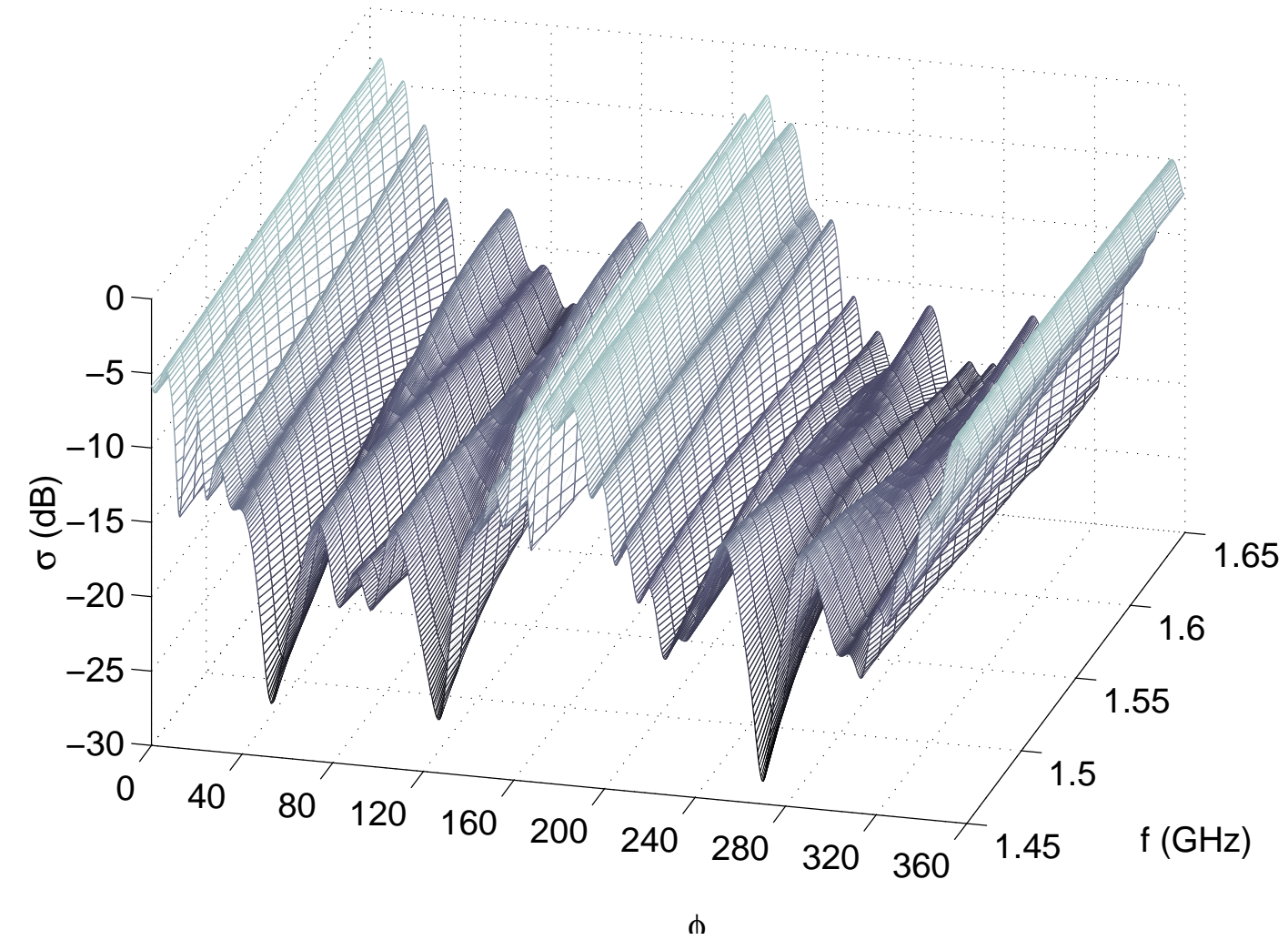

Figure 6: The monostatic Radar Cross Section computed in the frequency sweep.

\section{Conclusions}

In this paper we followed up on some of the issues in [6].

The straight forward extension to matrices with varying parameters was carried out. A similar theorem as in [6] was proved for this case in Theorem 1. In the numerical experiments it was demonstrated that exponential convergence rate is achieved in frequency if the Fast Multipole Method is used with Method of Moments.

For the case of electromagnetic plane wave scattering an estimate on the number of angles required before the remaining solutions are accurately predicted by MRI is given. The experiments validate this result.

Finally, an experiment on using MRI in both frequency and angle shows the additional benefit with this method. The number of matrix vector multiplication is reduced by a factor of about 60 compared to solving one right hand side at a time. The work is also reduced compared to only using MRI in one of the 


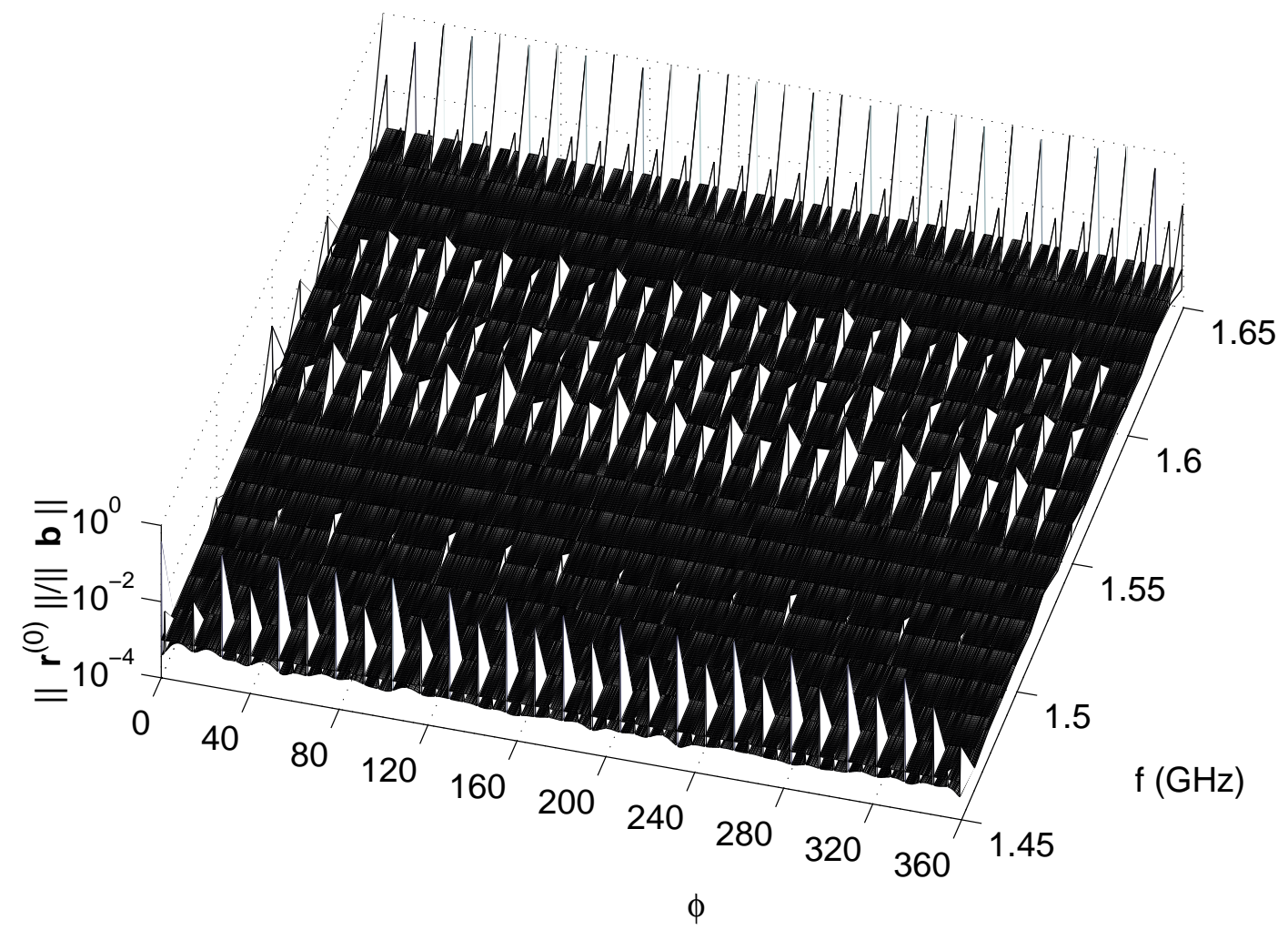

Figure 7: The residual of the initial guess computed in the frequency sweep.

two parameters. Since many of the matrix vector products are carried out in the initialization step of each frequency point, it would be worthwhile to find a more efficient way of doing the initializations. For certain classes of matrices the method suggested in [6] should be used. The Method of Moments requires a different approach. One possibility is to use some kind of interpolation, but it has to be done in a rigorous way in order to ensure that the residual estimates hold. Otherwise, more matrix vector multiplications are needed. This is a subject for further research.

\section{References}

[1] Milton Abramowitz and Irene A. Segun. Handbook of Mathematical Functions. Dover Publications Inc., New York, 1968.

[2] E. W. Cheney. Introduction to Approximation Theory. McGraw-Hill, New York, p. 60, 1966.

[3] Weng Cho Chew, Jian-Ming Jin, Eric Michielssen, and Jiming Song. Fast 
and Efficient Algorithms in Computational Electromagnetics. Artech House, Inc., Norwood, 2001.

[4] Ronald Coifman, Vladimir Rokhlin, and Stephen Wandzura. The fast multipole method for the wave equation: A pedestrian prescription. IEEE Transactions on Antennas and Propagation, 35(3):7-12, June 1993.

[5] Misha Kilmer, Eric Miller, and Carey Rappaport. QMR-based projection techniques for the solution of non-Hermitian systems with multiple right hand sides. SIAM Journal of Scientific Computing, 23(3):761-780, 2002.

[6] Per Lötstedt and Martin Nilsson. A minimal residual interpolation method for linear equations with multiple right hand sides. Technical Report 2002041, Department of Information Technology, Scientific Computing, Uppsala University, Dec. 2002. Accepted for publication (in revised form) in SIAM Journal of Scientific Computing.

[7] Martin Nilsson. A fast multipole accelerated block quasi minimum residual method for solving scattering from perfectly conducting bodies. In Proceedings of Antennas and Propagation Society International Symposium, volume 4, pages 1848-1851, Salt Lake City, Utah, USA, July 2000.

[8] Martin Nilsson. Iterative solution of Maxwell's equations in frequency domain. Licentiate thesis No. 2002-004, Department of Information Technology, Uppsala University, May 2002. Available at: http://www.it.uu.se/research/reports/lic/2002-004/.

[9] Andrew F. Peterson, Scott L. Ray, and Raj Mittra. Computational Methods for Electromagnetics. IEEE Press and Oxford University Press, New York, Oxford, Tokyo, Melbourne, 1998.

[10] Sadasiva. M. Rao, Donald. R. Wilton, and Allen. W. Glisson. Electromagnetic scattering by surfaces of arbitrary shape. IEEE Transactions on Antennas and Propagation, 30(3):409-418, May 1982.

[11] Youcef Saad and Martin H. Schultz. GMRES: A generalized minimal residual algorithm for solving nonsymmetric linear systems. SIAM J. Sci. Comput., 7:856-869, 1986.

[12] V. Simoncini and E. Gallopoulos. An iterative method for nonsymmetric systems with multiple right-hand sides. SIAM Journal of Scientific Computing, 16:917-933, 1995.

[13] V. Simoncini and E. Gallopoulos. Convergence properties of block GMRES and matrix polynomials. Lin. Alg. Appl., 247:97-119, 1996. 
[14] Jiming Song and Weng Cho Chew. Multilevel fast multipole algorithm for solving combined field integral equation of electromagnetic scattering. $\mathrm{Mi}$ crowave and Optical Technology Letters, 10(1):14-19, September 1995. 